\title{
Long-term air pollution exposure and incident dementia in American elderly population: a national cohort study (2000-2018)
}

Liuhua Shi ( $\square$ liuhua.shi@emory.edu )

Emory University https://orcid.org/0000-0001-8165-4644

Kyle Steenland

Emory University

Haomin Li

Emory University

Pengfei Liu

Georgia Institute of Technology

Yuhan Zhang

Emory University

Robert Lyles

Emory University

\section{Weeberb Requia}

Fundação Getúlio Vargas https://orcid.org/0000-0002-7564-3364

Sindana llango

University of Washington

Howard Chang

Emory University

Thomas Wingo

Emory University https://orcid.org/0000-0002-7679-6282

Rodney Weber

Georgia Institute of Technology

Joel Schwartz

Harvard University https://orcid.org/0000-0001-6168-378X

Article

Keywords:

Posted Date: May 26th, 2021

DOI: https://doi.org/10.21203/rs.3.rs-514522/v1 
License: (c) (i) This work is licensed under a Creative Commons Attribution 4.0 International License. Read Full License

Version of Record: A version of this preprint was published at Nature Communications on November 19th, 2021. See the published version at https://doi.org/10.1038/s41467-021-27049-2. 


\section{$1 \quad$ Long-term air pollution exposure and incident dementia in an American elderly 2 population: a national cohort study (2000-2018)}

4 Liuhua Shi ${ }^{* \# 1}$, Kyle Steenland ${ }^{\# 1}$, Haomin $\mathrm{Li}^{2}$, Pengfei Liu ${ }^{3}$, Yuhan Zhang ${ }^{2}$, Robert H. Lyles ${ }^{4}$, 5 Weeberb J. Requia ${ }^{5}$, Sindana D. Ilango ${ }^{6}$, Howard H. Chang ${ }^{1,4}$, Thomas Wingo ${ }^{7}$, Rodney J.

6 Weber $^{3}$, Joel Schwartz ${ }^{8}$

$8 \quad$ \# LS and KS contributed equally.

$9{ }^{1}$ Gangarosa Department of Environmental Health, Rollins School of Public Health, Emory University, Atlanta, GA

$10{ }^{2}$ Department of Epidemiology, Rollins School of Public Health, Emory University, Atlanta, GA

$11{ }^{3}$ School of Earth and Atmospheric Sciences, Georgia Institute of Technology, Atlanta, Georgia, USA

$12{ }^{4}$ Department of Biostatistics and Bioinformatics, Rollins School of Public Health, Emory University, Atlanta, Georgia, 13 USA

$14{ }^{5}$ School of Public Policy and Government, Fundação Getúlio Vargas, Brasília, Distrito Federal, Brazil

$15{ }^{6}$ Department of Epidemiology, School of Public Health, University of Washington, Seattle, Washington, USA

$16{ }^{7}$ Department of Neurology and Human Genetics, School of Medicine, Emory University, Atlanta, Georgia, USA

$17{ }^{8}$ Department of Environmental Health, Harvard T.H. Chan School of Public Health, Boston, Massachusetts, USA

19 Correspondence to: Liuhua Shi, Gangarosa Department of Environmental Health, Rollins School of Public 20 Health, Emory University, Atlanta, Georgia, USA

21 Telephone: (339)221-8486

22 E-mail: liuhua.shi@emory.edu 
27 Epidemiological evidence suggests air pollution exposure may increase risk of Alzheimer's

28 disease and related dementias (ADRD). However, previous U.S. studies have predominantly

29 focused on hospitalizations, which fails to fully capture ADRD. Here we constructed two national

30 population-based cohorts of those aged 65 and above from the Medicare Chronic Conditions

31 Warehouse (2000-2018), including doctor visits, to investigate the impact of long-term exposure

32 to ambient fine particulate matter $\left(\mathrm{PM}_{2.5}\right)$, nitrogen dioxide $\left(\mathrm{NO}_{2}\right)$, and ozone $\left(\mathrm{O}_{3}\right)$ on dementia

33 and $A D$ incidence, respectively. We identified $\sim 2.0$ million incident dementia cases

$34(N=12,233,371$; dementia cohort $)$ and $\sim 0.8$ million incident $A D$ cases $(N=12,456,447 ; A D$

35 cohort). Per interquartile range (IQR) increase in the 5-year average $\mathrm{PM}_{2.5}\left(3.2 \mu \mathrm{g} / \mathrm{m}^{3}\right), \mathrm{NO}_{2}$

36 (11.6 ppb), and warm-season $\mathrm{O}_{3}(5.3 \mathrm{ppb})$ over the past 5 years prior to diagnosis, the hazard

37 ratios (HRs) were 1.060 (95\% confidence interval [Cl]: 1.054, 1.066), 1.019 (95\% Cl: 1.012,

$381.026)$, and 0.990 (95\% Cl: 0.987, 0.993) for incident dementias, and 1.078 (95\% Cl: 1.070,

$391.086), 1.031(95 \% \mathrm{Cl}: 1.023,1.039)$, and $0.982(95 \% \mathrm{Cl}: 0.977,0.986)$ for incident $\mathrm{AD}$,

40 respectively, for the three pollutants. For both outcomes there was strong evidence of linearity in

41 concentration-response relationships for $\mathrm{PM}_{2.5}$ and $\mathrm{NO}_{2}$, suggesting the lack of a clear safe

42 threshold for these health-harmful pollutants. Our study suggests that exposures to $\mathrm{PM}_{2.5}$ and

$43 \mathrm{NO}_{2}$, but not $\mathrm{O}_{3}$, may increase the incidence of dementia and $\mathrm{AD}$. Improving air quality may

44 reduce the burden of $A D R D$ and promote healthy aging. 
46 Dementia is a major public health issue with substantial health and financial burden, affecting

47 more than 47 million people worldwide ${ }^{1}$. Alzheimer's disease (AD) contributes to about two-

48 thirds of dementia cases and is the sixth leading cause of death in the United States ${ }^{2}$. In

49 response to this devastating public health threat, the National Alzheimer's Project Act was

50 signed into law to overcome dementia, and the National Plan was launched with Goal 1 aiming

51 to prevent and effectively treat dementia (delay onset, slow progression) by $2025^{3}$. As there are

52 no disease-modifying treatments for the most common types of dementia, it is a top research

53 priority to identify modifiable risk factors for dementia that can be intervened on at the

54 population level.

There is growing evidence associating air pollution with neurodegenerative disease. A

56 systematic review by Peters et al. $(2019)^{4}$, found 9 longitudinal studies of air pollution and

57 Alzheimer's disease and related dementias (ADRD). Among them, 5 of 6 showed a positive

58 association between increased exposure to $\mathrm{PM}_{2.5}$ and dementia or AD; 4 of 4 showed an

59 association between $\mathrm{NO}_{2}$ and dementia or $\mathrm{AD}$, while 1 of 3 did so for ozone $\left(\mathrm{O}_{3}\right)$. Fu and Yung

$60(2020)^{5}$ published a review and meta-analysis of AD and air pollution, and found a 2-fold excess

61 risk of $A D$ for a $10 \mu \mathrm{g} / \mathrm{m}^{3}$ increase of $\mathrm{PM}_{2.5}$ among 6 studies, and no increased risk for $\mathrm{NO}_{2}$ in

62 four studies, nor for $\mathrm{O}_{3}$ in three studies. There have been several longitudinal studies since

63 these reviews, with the majority finding positive associations between air pollutants and either

64 dementia or $\mathrm{AD}^{6-14}$. A few of these studies examine the associations in US populations, and

65 these studies have almost exclusively used hospitalization as a measure of morbidity $6,7,11,13$. The

66 diagnosis of ADRD, however, likely occurs in doctor visits, and ADRD does not generally result

67 in hospitalizations. Thus, hospitalization records may not well represent either disease incidence

68 or prevalence, and likely leads to an underestimation of the number of cases. In addition, 
neuropathologic changes are known to occur many years prior to the diagnosis ${ }^{15}$, and the relevant time window in which air pollution might increase the risk of dementia or $A D$ is unclear.

To address these knowledge gaps in studying ADRD incidence in the US, here we constructed a national, population-based cohort study from Medicare data to investigate the

73 impact of long-term exposure to $\mathrm{PM}_{2.5}, \mathrm{NO}_{2}$, and $\mathrm{O}_{3}$ on dementia and $\mathrm{AD}$ incidence. To better

74 approximate disease incidence, we required a 5-year "clean" period without events of interest

75 and used all Medicare claims nationwide (2000-2018), including Medicare inpatient and

76 outpatient claims, carrier file (primarily doctor visits), skilled nursing facility, and home health-

77 care claims. We ascertained air pollution based on resident ZIP code, averaged over 5 years

78 prior to diagnosis, which was estimated from national spatiotemporal ensemble exposure 79 models.

\section{Results}

81 Study population characteristics. Table 1 provides descriptive information on the dementia cohort and $A D$ cohort. Both cohorts were followed after requiring a 5-year period without events

83 of interest to better capture disease incidence. There were 12.2 and 12.4 million people in the

84 dementia and AD cohorts, respectively (Table 1). Most of the studied subjects (78.5\% and

$8578.1 \%$ for dementia and AD, respectively) entered the cohorts between ages 65 and 74 . The

86 median follow-up was 7 years in both cohorts. $16.6 \%$ developed dementia ( 2.0 million cases),

87 and $6.5 \%$ developed $A D(\sim 0.8$ million cases). More than $90 \%$ were not eligible for Medicaid,

88 indicating that most were above the poverty level. A majority of the study population had a

89 comorbidity at some point during follow-up. 
Table 1. Descriptive statistics for the study population

\begin{tabular}{|c|c|c|c|c|}
\hline & \multicolumn{2}{|c|}{ Dementia cohort } & \multicolumn{2}{|c|}{ AD cohort } \\
\hline Variables & Number & $\%$ & Number & $\%$ \\
\hline Number of events & $2,025,130$ & 16.6 & 804,668 & 6.5 \\
\hline Number of total population & $12,233,371$ & 100 & $12,456,447$ & 100 \\
\hline Total person-years & $89,035,081$ & 100 & $93,278,266$ & 100 \\
\hline Median follow-up years & \multicolumn{2}{|l|}{7} & \multicolumn{2}{|l|}{7} \\
\hline \multicolumn{5}{|l|}{ Age at entry (years) } \\
\hline $65-74$ & $9,597,788$ & 78.5 & $9,734,481$ & 78.1 \\
\hline $75-114$ & $2,635,583$ & 21.5 & $2,721,966$ & 21.9 \\
\hline \multicolumn{5}{|l|}{ Sex } \\
\hline Male & $5,023,879$ & 41.1 & $5,107,942$ & 41.0 \\
\hline Female & $7,209,492$ & 58.9 & $7,348,505$ & 59.0 \\
\hline \multicolumn{5}{|l|}{ Race } \\
\hline White & $11,023,202$ & 90.1 & $11,214,287$ & 90.0 \\
\hline Black & 649,081 & 5.3 & 666,619 & 5.4 \\
\hline Other & 561,088 & 4.6 & 575,541 & 4.6 \\
\hline \multicolumn{5}{|l|}{ Medicaid Eligibility } \\
\hline Dual-Eligible & 800,139 & 6.5 & 852,499 & 6.8 \\
\hline Non-dual Eligible & $11,433,232$ & 93.5 & $11,603,948$ & 93.2 \\
\hline \multicolumn{5}{|l|}{ Comorbidity } \\
\hline Diabetes & $4,433,314$ & 36.2 & $4,590,000$ & 36.8 \\
\hline Hypertension & $10,273,506$ & 84.0 & $10,502,180$ & 84.3 \\
\hline Stroke & $1,991,730$ & 16.3 & $2,137,239$ & 17.2 \\
\hline Heart failure & $3,388,540$ & 27.7 & $3,598,028$ & 28.9 \\
\hline No comorbidities ${ }^{\mathrm{a}}$ & $1,642,674$ & 13.4 & $1,865,751$ & 15.0 \\
\hline \multicolumn{5}{|l|}{ Air pollutants ${ }^{\mathrm{b}}$} \\
\hline Annual $\mathrm{PM}_{2.5}\left(\mu \mathrm{g} / \mathrm{m}^{3}\right)$ & \multicolumn{2}{|c|}{$9.3(3.2)$} & \multicolumn{2}{|c|}{$9.3(3.2)$} \\
\hline Annual $\mathrm{NO}_{2}(\mathrm{ppb})$ & \multicolumn{2}{|c|}{$17.1(11.6)$} & \multicolumn{2}{|c|}{$17.1(11.6)$} \\
\hline Warm-season $\mathrm{O}_{3}(\mathrm{ppb})$ & \multicolumn{2}{|c|}{$42.6(5.3)$} & \multicolumn{2}{|c|}{$42.6(5.3)$} \\
\hline
\end{tabular}

Note: a means none of the above comorbidities; ${ }^{b}$ presented as mean concentration (interquartile range).

Air pollution levels. The average annual level of $\mathrm{PM}_{2.5}$ of cohort participants during the study period, $9.3 \mu \mathrm{g} / \mathrm{m}^{3}$, was below the US EPA standard of $12 \mu \mathrm{g} / \mathrm{m}^{3} ; \mathrm{NO}_{2}$ levels were considerably below the EPA annual standard of $\mathrm{NO}_{2}$ of $53 \mathrm{ppb}$. The annual warm-season average $\mathrm{O}_{3}$ was 42.6 ppb. EPA does not have a standard for annual warm-season $\mathrm{O}_{3}$. As a reference, the EPA standard for daily maximum of 8-hour average $\mathrm{O}_{3}$ is $75 \mathrm{ppb}$. We examined warm-season $\mathrm{O}_{3}$, because $\mathrm{O}_{3}$ is more readily formed in the warm season ${ }^{16}$, and this metric is often used in longterm epidemiological studies ${ }^{17}$. Figure 1 shows the distribution of the three pollutants across the 
102 US during our study period, as estimated by the exposure models used in our analysis. $\mathrm{PM}_{2.5}$ is

103 highest in the eastern US, $\mathrm{O}_{3}$ in the West, and $\mathrm{NO}_{2}$ (largely produced by traffic) in urban

104 centers. Further detail on exposure levels can be found in Supplementary Table S1. The three

105 pollutants in our data were only modestly correlated. The Pearson correlations between

106 pollutants (average exposure within the past 5 years) were as follows: $\mathrm{PM}_{2.5}$ and $\mathrm{O}_{3} 0.22, \mathrm{NO}_{2}$

107 and $\mathrm{O}_{3} 0.19$, and $\mathrm{NO}_{2}$ and $\mathrm{PM}_{2.5} 0.39$.

108 Health effect estimates. Figure 2 provides the main study results from the Cox proportional

109 hazards models stratified by individual characteristics, adjusting for neighborhood-level

110 socioeconomic status (SES), behavioral risk factors, health care capacity variables, and residual

111 temporal and spatial trends (see Methods). An interquartile range (IQR) increase in the 5-year

112 average of the annual $\mathrm{PM}_{2.5}\left(3.2 \mu \mathrm{g} / \mathrm{m}^{3}\right)$ in the 5 years prior to diagnosis was associated with an

113 increased risk of dementia (HR=1.061, 95\% Cl: $1.056,1.067)$ in single pollutant models, which

114 changes little in models with other pollutants. An IQR increase in 5-year average $\mathrm{NO}_{2}(11.6 \mathrm{ppb})$

115 is associated with an $\mathrm{HR}$ of $1.035(95 \% \mathrm{Cl}: 1.028,1.042)$ in single pollutant models, dropping to

$1161.019(95 \% \mathrm{Cl}: 1.012,1.026)$ in multi-pollutant models. An IQR increase in the 5-year average of

117 warm-season $\mathrm{O}_{3}(5.3 \mathrm{ppb})$ has little effect on dementia rates, with HRs of 1.002 (95\% Cl: 0.998,

$1181.005)$ in single pollutant models and $0.990(95 \% \mathrm{Cl}: 0.987,0.993)$ in multi-pollutant models.

119 The findings for AD have a similar pattern to those for dementia, but the hazard ratios

120 are higher per IQR increase, being $1.078(95 \% \mathrm{Cl}: 1.071,1.086)$ for $\mathrm{PM}_{2.5}, 1.050(95 \% \mathrm{Cl}: 1.042$,

121 1.059) for $\mathrm{NO}_{2}$, and $0.999(95 \% \mathrm{Cl}: 0.995,1.003)$ for $\mathrm{O}_{3}$ assessing each pollutant individually.

122 After adjusting for co-pollutants, the effect estimates were similar for $\mathrm{PM}_{2.5}(\mathrm{HR}=1.078,95 \% \mathrm{Cl}$ :

$1231.070,1.086)$ and attenuated for $\mathrm{NO}_{2}(\mathrm{HR}=1.031,95 \% \mathrm{Cl}: 1.023,1.039)$, while $\mathrm{O}_{3}$ is slightly

124 protective (HR=0.982, 95\%Cl: $0.977,0.987)$. 
(a)

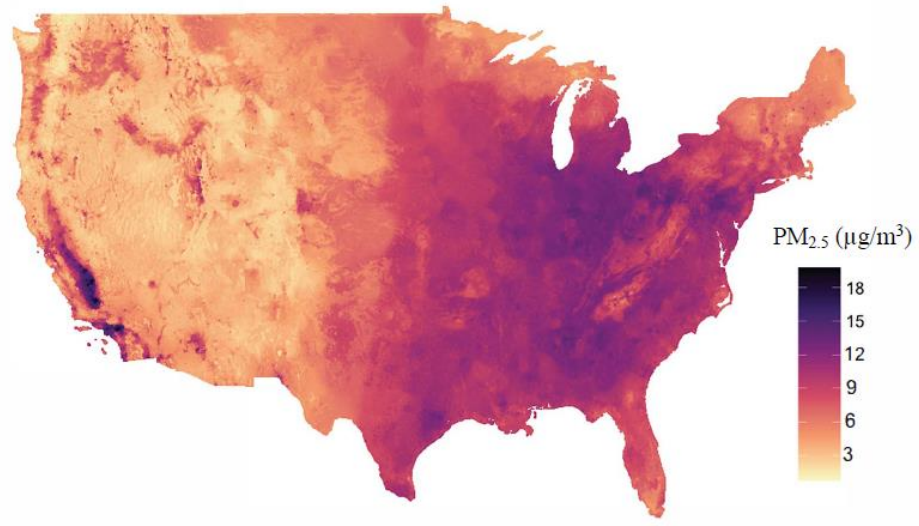

(b)

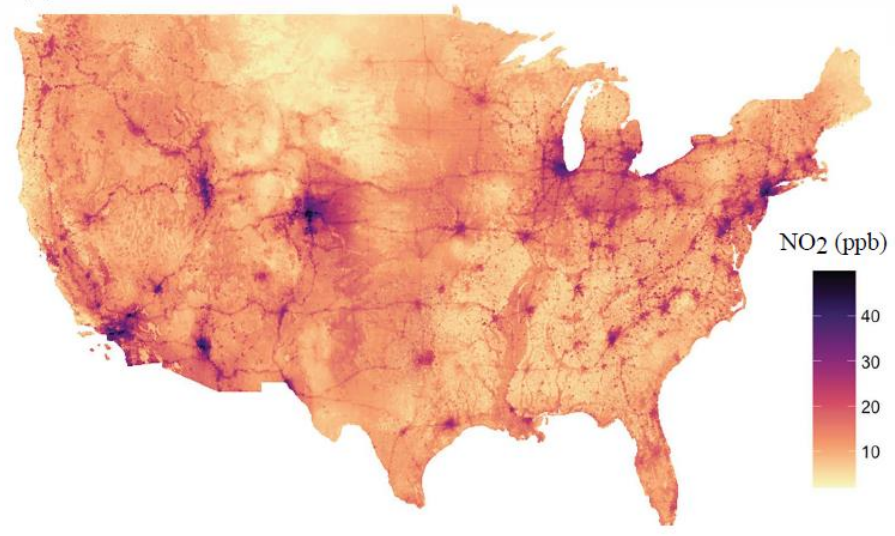

(c)

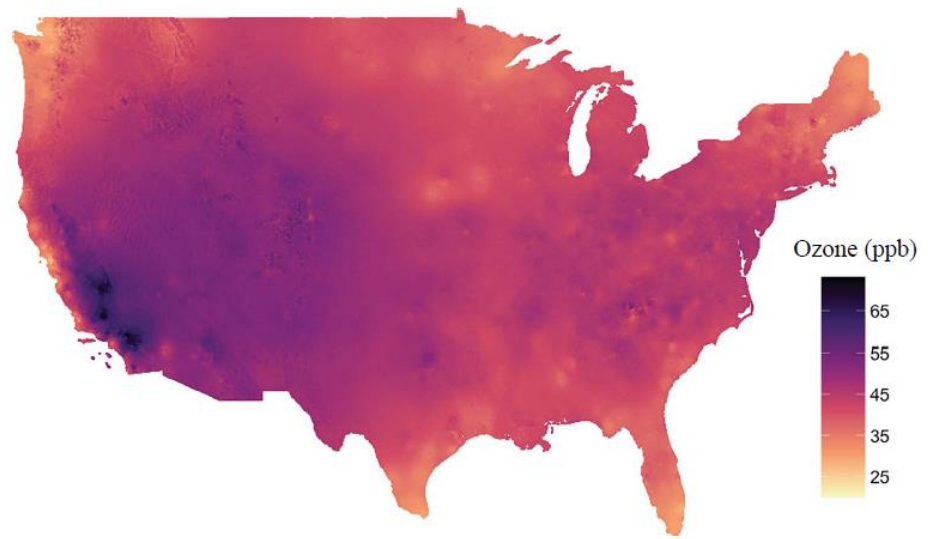

127 Fig.1| Average concentrations of (a) annual $\mathrm{PM}_{2.5}\left(\mu \mathrm{g} / \mathrm{m}^{3}\right)$, (b) annual $\mathrm{NO}_{2}(\mathrm{ppb})$, and (c) warm-season $128 \mathrm{O}_{3}(\mathrm{ppb})$ across the contiguous United States over the study period. 
(a) Dementia

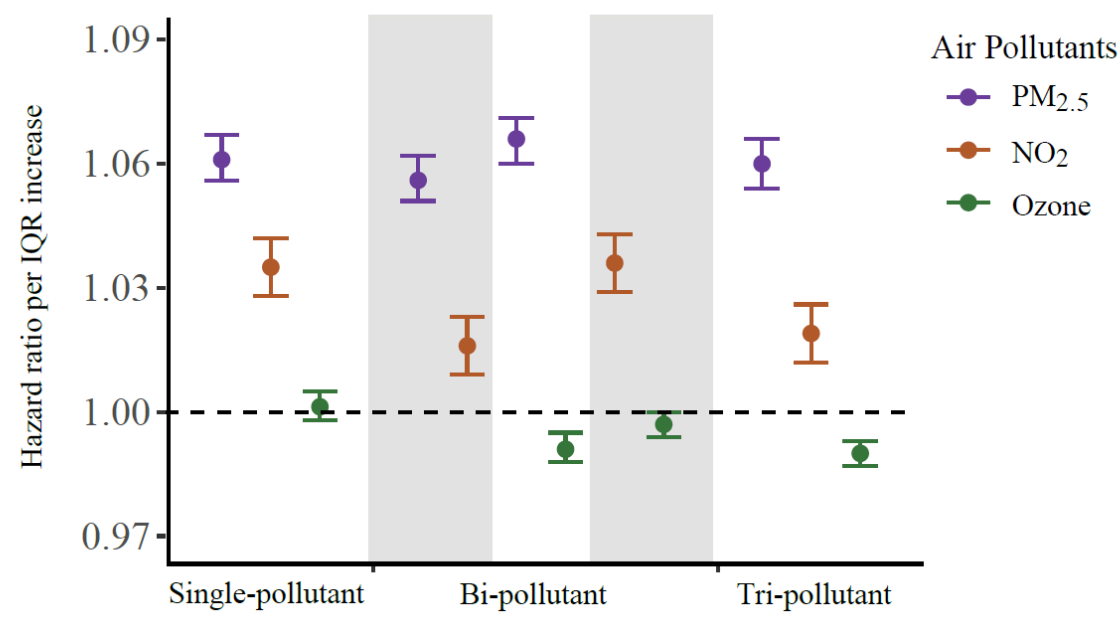

(b) Alzheimer's disease

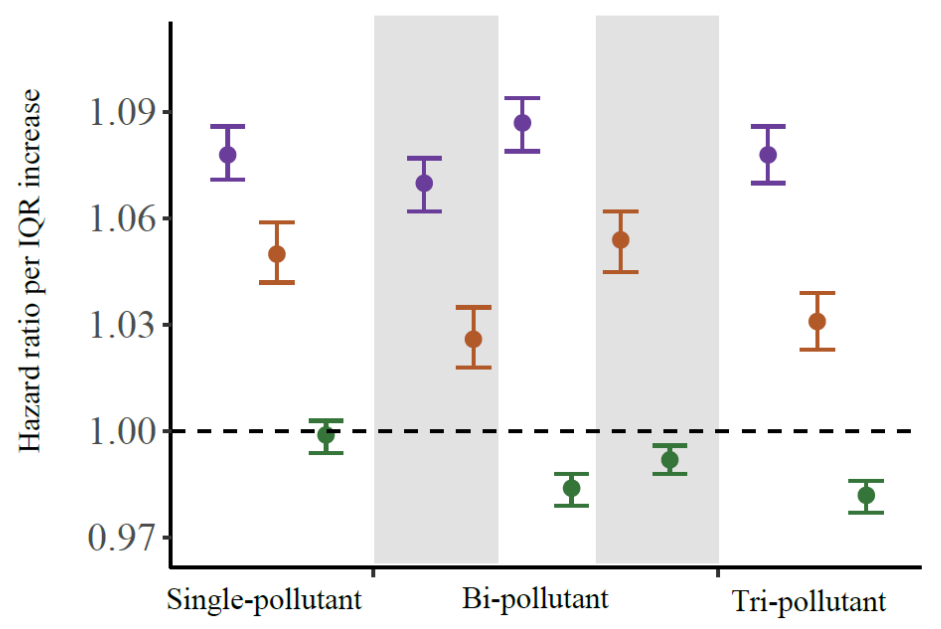

Fig.2 Hazard ratios of dementia or Alzheimer's disease (AD) associated with per IQR increase in annual $\mathrm{PM}_{2.5}(\mathrm{a})$, or annual $\mathrm{NO}_{2}(\mathrm{~b})$, or warm-season $\mathrm{O}_{3}$ (c) concentration. The estimated hazard ratios were obtained using single pollutant. bi-pollutant, and tri-pollutant models.

Concentration-response relationships. Figure 3 presents penalized spline curves for the

136 three pollutants, derived from the tri-pollutant models. The concentration-response (C-R)

137 relationships for $\mathrm{PM}_{2.5}$ are approximately linear for both dementia and $\mathrm{AD}$ across the exposure

138 distribution, although for $A D$ there is a suggestion of a steeper slope below $8 \mu \mathrm{g} / \mathrm{m}^{3}$. For $\mathrm{NO}_{2}$,

139 the C-R curves for dementia and AD are linear for low concentrations ( $<25 \mathrm{ppb})$, and then level

140 off for higher concentrations. The curves for $\mathrm{O}_{3}$ are essentially flat for both endpoints until high,

141 and rarely occurring concentrations. These results suggest that the adverse effects of $\mathrm{PM}_{2.5}$ and 
$142 \mathrm{NO}_{2}$ on dementia or $\mathrm{AD}$ are at least retained, if not strengthened, at low levels of air pollution

143 exposure (e.g., below the WHO air quality guidelines: $\mathrm{PM}_{2.5} \leq 10 \mu \mathrm{g} / \mathrm{m}^{3}, \mathrm{NO}_{2} \leq 20 \mathrm{ppb}$ ). Across

144 the 0.5 th to 99.5th percentile of the exposure distribution, $\mathrm{PM}_{2.5}$ shows the strongest effect on

145 dementia or AD among all pollutants.

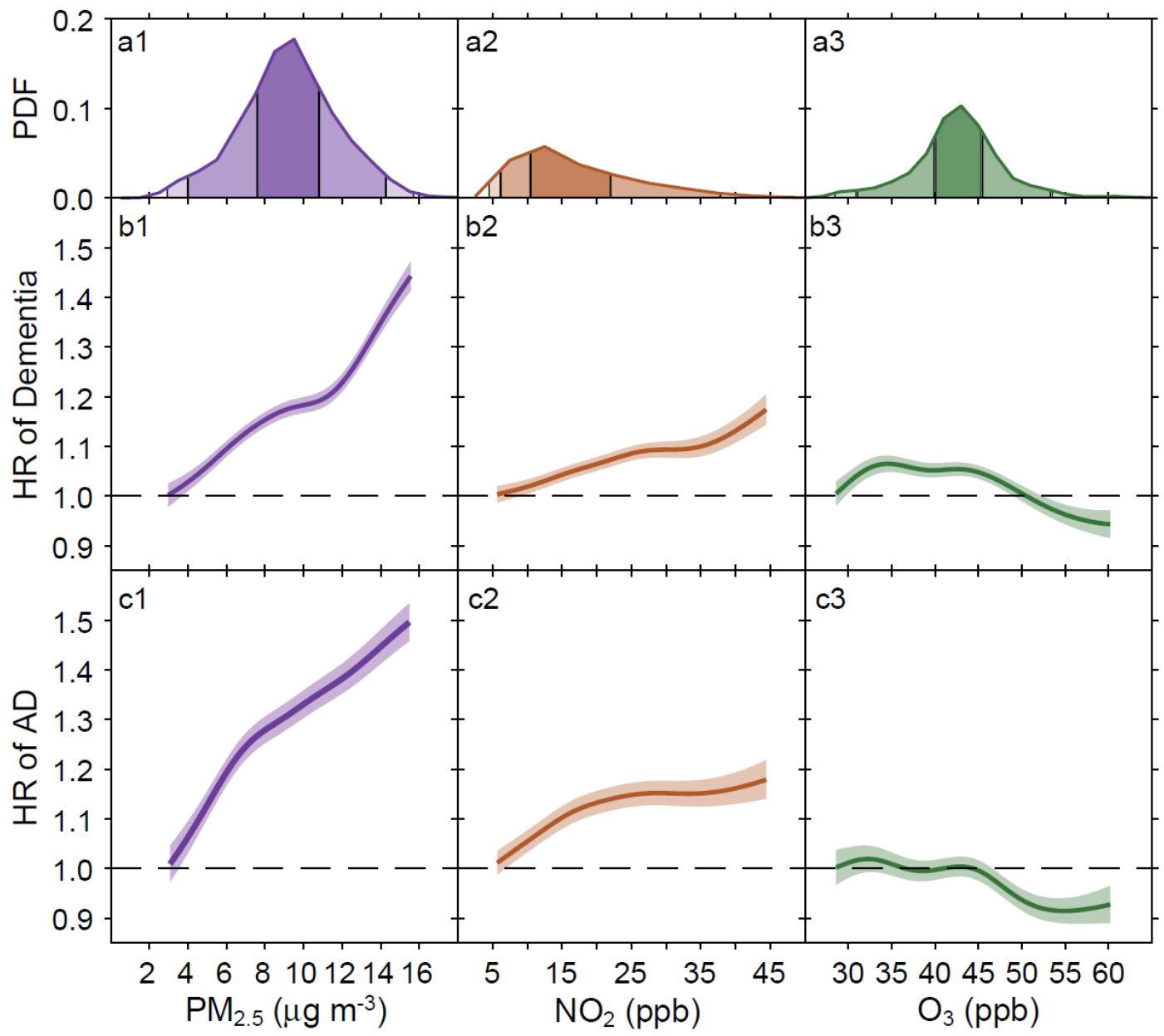

147 Fig.3| (a) Probability distribution functions (PDF) of long-term $\mathrm{PM}_{2.5}, \mathrm{NO}_{2}$, and $\mathrm{O}_{3}$ exposures; concentration-response relationships between each pollutant and (b) dementia and (c) Alzheimer's disease (AD). The concentration-response curves, derived from the tri-pollutant models, are shown for the concentration ranges between 0.5 th to 99.5 th percentiles of the pollutants, i.e. with $1 \%$ poorly constrained extreme values excluded. Shading areas (from the darkest to the lightest) in panels (a) represent pollutant concentration ranges of the IQR (i.e., 25th to 75th percentiles), 95\% (2.5th to 97.5th), and $99 \%$ (0.5th to 99.5 th), respectively. 
154 Effect modifications. We examined 5 potential effect modifiers (gender, race

155 (white/black/other), Medicaid eligibility, population density (quartiles), and age $(<75, \geq 75)$. Figure

1564 shows hazard ratios in each subgroup, based on the interaction term between $\mathrm{PM}_{2.5}$ or $\mathrm{NO}_{2}$,

157 and the potential effect modifier. Most marked results were seen for an increased hazard of

158 dementia and $\mathrm{AD}$ for blacks vs. whites in relation to both $\mathrm{PM}_{2.5}$ and $\mathrm{NO}_{2}$; a similar pattern was

159 found for those eligible for Medicaid. At the same time, those living in the rural areas (i.e. lowest

160 quartile of population density) were found to have a notably lower association between both

161 dementia and $\mathrm{AD}$ and both $\mathrm{PM}_{2.5}$ and $\mathrm{NO}_{2}$. All three of these effect modifiers may reflect a

162 general pattern of increased susceptibility to the effects of $\mathrm{PM}_{2.5}$ and $\mathrm{NO}_{2}$ among those of lower

163 SES and lower education (similar effect modification was seen in Ailshire et al. $2017^{18}$ and

164 Ailshire et al. $2021^{19}$, but not seen in Mortamais et al. $2021^{12}$ or in Cerza et al. $2019^{20}$ ).

165 Regarding age, those less than 75 had a markedly stronger association between dementia and

166 both $\mathrm{PM}_{2.5}$ and $\mathrm{NO}_{2}$, while the association was stronger between $\mathrm{AD}$ and $\mathrm{NO}_{2}$ among those

167 older than 75. Finally, we found little evidence of an interaction between pollution and gender in

168 relation to dementia or $\mathrm{AD}$. Figure $\mathrm{S} 1$ shows the results for $\mathrm{O}_{3}$, with relatively few hints of effect

169 modification and all subgroup-specific estimated hazard ratios below one. 

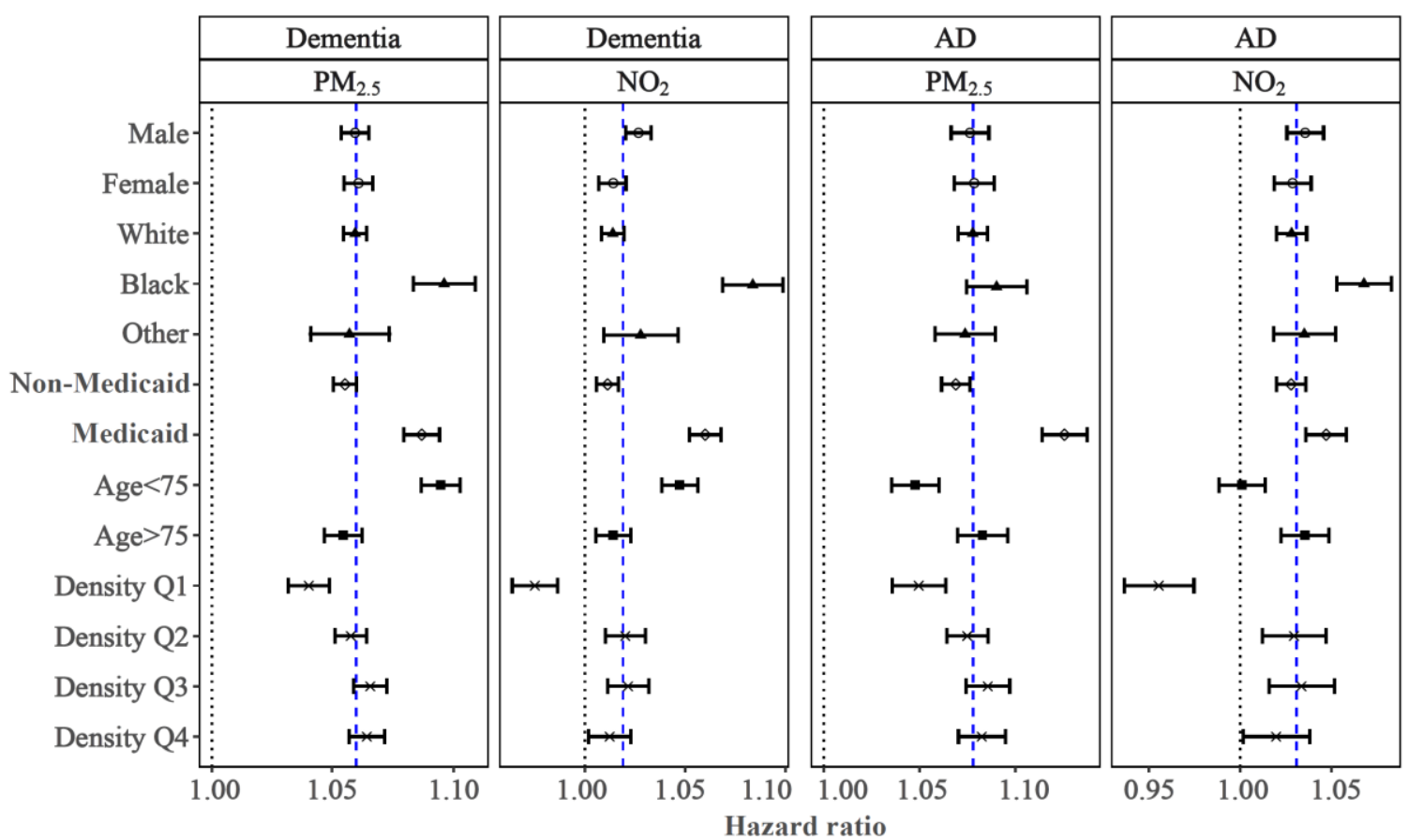

Fig.4| Effect modifications by sex, race, Medicaid eligibility, age, and population density. Results represent the hazard ratios of dementia or Alzheimer's disease (AD), from the tri-pollutant models, per IQR increase in 5-year average $\mathrm{PM}_{2.5}$ or $\mathrm{NO}_{2}$. The blue dashed lines indicate the overall effect estimates for all groups. "Density Q1-Q4" denotes quartiles of population density, i.e., low population density, lowmedium population density, medium-high population density, and high population density.

Attributable fraction. The strongest relationship we found with both endpoints was for $\mathrm{PM}_{2.5}$

177 among the three pollutants. If the US PM 2.5 levels could be lowered by $3.2 \mu \mathrm{g} / \mathrm{m}^{3}$, which is the

178 IQR, then the attributable fraction of dementia and AD due to current exposure levels, based on

179 our main results from tri-pollutant models assuming a linear relationship, would be $6 \%$ and $7 \%$ respectively. Namely, an estimated $6 \%$ of dementia cases and $7 \%$ of $A D$ cases would be avoided if $\mathrm{PM}_{2.5}$ levels decreased by $3.2 \mu \mathrm{g} / \mathrm{m}^{3}$, which is approximately the difference between our large cities like New York and Chicago and smaller cities like Portland, Buffalo, or Baltimore ${ }^{21}$.

Sensitivity analysis. Associations between long-term exposure to $\mathrm{PM}_{2.5}, \mathrm{NO}_{2}, \mathrm{O}_{3}$ and dementia or AD were robust to a series of sensitivity analyses. First, a more strict "clean period" 
187 yielded results similar to the main analyses (Supplementary Table S2). Second, based on this

188 new subcohort (with 10-year clean period), the use of alternative exposure windows (annual

189 exposure $10,5,1$, or 0 years prior to disease diagnosis, i.e., lags $10,5,1$, or 0 ) all support a

190 positive association with $\mathrm{PM}_{2.5}$ and $\mathrm{NO}_{2}$, but not $\mathrm{O}_{3}$, though $\mathrm{HRs}$ varied in magnitude

191 (Supplementary Table S2). For both outcomes, associations with $\mathrm{PM}_{2.5}$ and $\mathrm{NO}_{2}$ were

192 attenuated with increasing lag periods. Third, the observed associations with dementia or AD

193 were not mediated by nor modified by comorbidities, such as diabetes, hypertension, stroke,

194 and heart failure (Supplementary Table S3). Lastly, we assessed the effect of possible outcome

195 misclassification in two ways, one via using a linear regression model based on rates, and the

196 other based on prior estimates of Medicare sensitivity and specificity and estimating the true

197 number of cases within strata. Both methods support the findings from our main analysis i.e.,

198 long-term exposure to $\mathrm{PM}_{2.5}$ and $\mathrm{NO}_{2}$, but not $\mathrm{O}_{3}$, were significantly associated with an

199 increased incidence of dementia and AD; both also suggest that misclassification has somewhat

200 biased our findings to the null (Supplementary Tables S4 and S5).

\section{Discussion}

202 We found elevated hazard ratios for both dementia and $A D$ in relation to $P M_{2.5}$, and less

203 markedly to $\mathrm{NO}_{2}$, while hazard ratios for warm-season $\mathrm{O}_{3}$ were not elevated. We did this study

204 in a large US cohort (12 million), with national coverage, and including non-urban areas. For

205 both $\mathrm{PM}_{2.5}$ and $\mathrm{NO}_{2}$, we found a larger effect on $\mathrm{AD}$ compared to dementia, which may reflect

206 that fact that dementia includes a wide range of diseases with distinct etiologies, some of which

207 may be unrelated to air pollution, while $A D$ is a subset of dementia and a single disease, for

208 which we found a stronger association. We also found that shorter time windows between

209 exposure $\left(\mathrm{PM}_{2.5}\right.$ or $\left.\mathrm{NO}_{2}\right)$ and disease showed higher effect estimates, and we posit that it

210 implies an acceleration of an existing process (i.e., accelerating cognitive decline which was

211 already well developed). Moreover, our diagnosis free period requirement provides reasonable 
212 assurance that we are looking at incidence, and use of physician's visits, nursing home data,

213 etc. to ascertain diagnosis avoids missing large numbers of cases, possibly not missing at

214 random, which likely occurs in studies using diagnoses based on hospital admission records.

Some of our models showed a protective effect of $\mathrm{O}_{3}$. However, when we compare

216 results in Figure 2, we see that in single pollutant models the effect estimate for $\mathrm{O}_{3}$ was null,

217 while in bi-pollutant models with either $\mathrm{PM}_{2.5}$ or $\mathrm{NO}_{2}$, the effect size for $\mathrm{O}_{3}$ was pushed below

218 the null (albeit not significantly) and only in the tri-pollutant model was it protective at the

219 conventional 0.05 level. Moreover, in the bi-pollutant models with $\mathrm{O}_{3}$, the effect sizes for $\mathrm{PM}_{2.5}$

220 and $\mathrm{NO}_{2}$ increased from their level in the single pollutant models. We interpret this as evidence

221 that there is no effect of $\mathrm{O}_{3}$, and the protective effect seen in the tri-pollutant model may be due

222 to collinearity.

223 Our results are broadly consistent with developing literature, which shows relatively

224 consistent effects for $\mathrm{PM}_{2.5}$ and $\mathrm{NO}_{2}$, but less consistent for $\mathrm{O}_{3}$. We observed an $\mathrm{HR}$ of 1.06 for

225 dementia and an HR of 1.08 for $A D$ per $3.2 \mu \mathrm{g} / \mathrm{m}^{3}$ increase in annual $\mathrm{PM}_{2.5}$ in single-pollutant

226 models, i.e., equivalent to an $\mathrm{HR}$ of 1.10 and an $\mathrm{HR}$ of 1.13 per $5 \mu \mathrm{g} / \mathrm{m}^{3}$ increase in $\mathrm{PM}_{2.5}$.

227 These values can be compared with our previous Medicare cohort study using hospitalizations ${ }^{7}$,

228 reporting an HR of 1.06 for dementia and an HR of 1.17 for AD per $5 \mu \mathrm{g} / \mathrm{m}^{3}$ increase in annual

$229 \mathrm{PM}_{2.5}$. A cohort study conducted in Ontario, Canada by Chen et al. $(2017)^{22}$ simultaneously

230 accessed the effects of $\mathrm{PM}_{2.5}, \mathrm{NO}_{2}$, and $\mathrm{O}_{3}$ on dementia risks, and they also found significant

231 associations with $\mathrm{PM}_{2.5}$ and $\mathrm{NO}_{2}$, but not $\mathrm{O}_{3}$. Recent 2018 and 2020 Lancet Commission

232 overviews of modifiable environmental agents associated with disease noted a possible

233 association between air pollutants and dementia, but noted the evidence still preliminary ${ }^{1,23}$.

234 The epidemiologic findings are supported by brain imaging and toxicologic studies.

235 Regarding brain imaging, Shaffer et al. (2021) ${ }^{24}$ have found associations between $\mathrm{PM}_{2.5}$ and AD 
236 neuropathology upon autopsy, while Laccarino et al. $(2020)^{25}$ found an association between

$237 \quad \mathrm{PM}_{2.5}$ and positive positron emission tomography (PET) scans for amyloid. Younan et al.

$238(2020)^{26}$ followed 1000 women and found increased cognitive decline on immediate

239 memory/new learning, and increased MRI-determined risk for future AD using a

240 neuroanatomical risk score. These recent findings support earlier neuroanatomical associations

241 found by others ${ }^{27,28}$. Toxicological studies support several plausible biological mechanisms.

$242 \mathrm{PM}_{2.5}$ has been consistently linked to oxidative stress, neuroinflammation, systemic

243 inflammation, and all of which, in turn, have been reported as key pathways to AD

244 pathogenesis ${ }^{27,29,30}$. Magnetite nanoparticles from combustion processes have been discovered

245 in the human brain, indicating that particles from urban air pollution can reach the blood-brain

246 barrier (e.g. through interacting with dysfunctional cell) ${ }^{31}$.

Our data suggest that lowering air pollution would have a meaningful reduction on $A D$ and dementia that, when applied to the US population, would be an important tool in the fight against dementia and AD. Assuming these associations are causal, our findings suggest that about $6 \%$ of dementia cases and $7 \%$ of $A D$ cases would be avoided if $P M_{2.5}$ levels decreased by $3.2 \mu \mathrm{g} / \mathrm{m}^{3}$.

251 It should be noted that - assuming our findings are generalizable to other parts of the world - the

252 potential decrease in the burden of $A D$ with lowered air pollution could be greater, considering 253 that the average annual $\mathrm{PM}_{2.5}$ level worldwide in 2015 was estimated at $42 \mu \mathrm{g} / \mathrm{m}^{3}{ }^{32}$

254 Our study has several strengths. To our knowledge, this is the first nationwide, 255 population-based cohort study that focuses on the simultaneous health effects of $\mathrm{PM}_{2.5}, \mathrm{NO}_{2}$, 256 and $\mathrm{O}_{3}$ on dementia and AD. The large sample size gives us ample power to detect effects even 257 though they are small, which is often the case in environmental studies. Second, the use of 258 Medicare claims data that include doctor's visits rather than restricting the data to 259 hospitalizations is likely to include many more cases, given that many cases are never 260 hospitalized, and also cases which are diagnosed earlier and hence better reflect incidence. 
261 Evidence can be found by comparing recent data in another paper about dementia and AD

262 hospitalization in Medicare data, ${ }^{7}$ to the data in the current paper. To allow for a fair comparison,

263 we used the same inclusion/exclusion criteria and restricted to the same time period (2000-

264 2016) and geographic region (i.e. the lower 48 states), and we found that using just

265 hospitalization missed nearly $90 \%$ of dementia cases and $60 \%$ of AD cases, compared to using

266 our current data including doctor's visits (Supplementary Table S6). Third, we used a

267 conservative method by requiring a 5-year "clean" period and restricting analysis to subjects

268 with continuous enrollment in Medicare FFS, and Part A (hospital insurance) and Part B

269 (medical insurance) programs throughout the study period, which can ensure that cases were

270 newly diagnosed and thus better approximate incidence. Lastly, we were able to control for a

271 large number of individual- and neighborhood-level covariates. Inclusion of comorbidities had

272 negligible effect on our results, suggesting that they are unlikely mediators in our studied

273 associations. However, a formal mediation analysis would be important to confirm these

274 findings.

275 Despite these advantages, some key limitations should be noted. One limitation, typical

276 of using administrative records to identify disease, is potential misclassification of outcome. AD

277 cases in our database represented only about $40 \%$ of the dementia cases, suggesting important

278 under-ascertainment of $A D$, given that $A D$ represents approximately $60-80 \%$ of dementia

279 cases $^{2}$. This percentage is quite similar to the findings of Goodman et al. (2017) $)^{33}$, who found

280 that AD represented $44 \%$ of all dementia diagnoses in Medicare data in 2013, including both

281 hospitalizations and doctor visits. It is likely that a large number of our dementia cases, who

282 show no AD diagnosis in Medicare, actually had AD, but physicians did not feel confident to

283 make the more specific diagnosis. This is supported by the findings of Taylor et al. (2009), who

284 compared Medicare data to clinical diagnoses considered as the gold standard, and found that

285 the sensitivity of dementia was 0.85 but was considerably lower, 0.65 , for AD. 
We have assumed that outcome misclassification is non-differential (conditionally

287 independent of exposure to air pollutants, conditional on confounders); there are no data

288 indicating otherwise. We have conducted two types of sensitivity analyses to adjust for such

289 misclassification of classifying dementia or AD cases as without dementia or AD (false negative,

290 or 1-sensitivity), and the misclassification of non-dementia, non-AD subjects to one of the

291 diseases (false positive, or 1-specificity). Both these methods of adjustment for false negative

292 and false positives were in agreement that our results were likely to under-estimate the true

293 hazard ratios for $\mathrm{PM}_{2.5}$ and $\mathrm{NO}_{2}$ for both dementia and $\mathrm{AD}$.

Another limitation of our study is the potential for exposure error, although the exposure 295 prediction model we used has excellent predictive accuracy ${ }^{34-36}$. Using larger scale ambient air 296 pollutions assigned to individuals has been shown to have a net bias towards the null, 297 consistent with non-differential measurement error, which reflects to some degree classical type 298 of error ${ }^{37-39}$. In addition, our study is subject to unmeasured and residual confounding. While we 299 were able to control for a number of potential confounders at the neighborhood level, we had no 300 individual-level data on SES and education, a limitation implying some mismeasurement of 301 confounders, which may have biased our results (moderately, given that these unmeasured 302 confounders are not likely to act as very strong risk factors for dementia), in an unknown 303 direction. Furthermore, we only studied the Medicare FFS population who enrolled in both Part A 304 and Part B programs, precluding generalizability to the entire US elderly population.

\section{Implications for future research}

Our study provides clear evidence that long-term exposure to $\mathrm{PM}_{2.5}$ mass is significantly 307 associated with increased ADRD incidence and lowering air pollution potentially has an 308 important public health effect. Future studies of air pollution and dementia in low-to-middle309 income countries (LMIC) of which there are few, will be important. Understanding the potential 
310 bias and unmeasured confounding, given the limitations of observational studies, is

311 encouraged. Examining the role of specific pollutant components in ADRD may also be

312 important, because different components of $\mathrm{PM}_{2.5}$ (e.g., metals, elemental carbon, organic

313 carbon, sulfate, and nitrate) and different sources of $\mathrm{PM}_{2.5}$ (e.g., traffic, industrial, cooking, and

314 biomass burning) may have different neurotoxicities. Better understanding of component-

315 specific and source-specific effects of $\mathrm{PM}_{2.5}$ on ADRD could inform pollution control policies on

316 specific sources.

\section{Methods}

\section{Study Population}

319 Data were drawn from the Medicare denominator file and the Medicare Chronic Conditions Warehouse

320 (CCW), both from the Centers for Medicare and Medicaid Services (CMS). The denominator file contains 321 enrollment records for each Medicare beneficiary in each year, including age, sex, race, Medicaid 322 eligibility (a proxy for socioeconomic status - SES), the date of death, and ZIP code of residence. CCW 323 provides the date of first occurrence with a dementia or AD diagnosis code across the available Medicare 324 claims. Based on these two Medicare databases, we constructed an open cohort including all Medicare 325 beneficiaries aged 65 and over who were always enrolled (1) in Medicare Fee-for-Service (FFS) program; 326 and (2) in both Medicare Part A (hospital insurance) and Part B (medical insurance) in the contiguous

327 United States between 2000 and 2018. These criteria excluded those with any time in Medicare 328 Advantage (HMO) over the study period since claim records are not available for these beneficiaries and 329 excluded those only enrolled in Medicare Part A or Part B. If we relaxed these restrictions to broaden the 330 cohort, the chance of missing dementia or AD cases among those additional people brought into the 331 analysis would be high.

332 We created separate datasets for dementia and AD. For each cohort, we further required a "clean" period 333 of 5 years after enrollment, during which there were no diagnosis codes for dementia or AD. By removing 334 potentially prevalent cases in their first five years of follow-up, a diagnosis after that "clean" period more 
likely approximates "incidence". We considered that 5 years was a reasonable period to ensure that a person truly was not demented prior to the Medicare diagnosis; however, we also explored a 10-year

337 clean period in sensitivity analyses. Therefore, study subjects entered the cohort on January 1 st of the

338 year following the "clean" period and were followed until first diagnosis of the outcome of interest across

339 all Medicare claims, death, or end of follow-up. We excluded this 5-year clean period from follow-up time

340 to avoid immortal time bias. This study was approved by the Institutional Review Board of Emory

341 University and a waiver of informed consent was granted.

Outcome classification

343 The primary outcomes of interest for this study were time to 1) all-cause dementia and 2) AD subtype.

344 CCW includes pre-defined indicators for dementia and $A D$, which are identified using an algorithm that 345 incorporates information from all available Medicare claims (such as inpatient and outpatient claims,

346 Carrier file, skilled nursing facility, and home health-care claims) indicating that an individual was

347 diagnosed with dementia or AD (Chronic condition algorithms $2015^{40}$ ). CCW provides the date of first 348 occurrence with a dementia or AD diagnosis code. In the dementia cohort, the outcome dementia was 349 defined as the first occurrence of a diagnosis code of dementia, while for the AD cohort, AD was defined 350 as either 1) the first occurrence of a diagnosis code of AD with no prior diagnosis of dementia, or the first 351 occurrence of a diagnosis code of dementia when there was a subsequent diagnosis code of AD (under 352 the supposition that the original dementia diagnosis was probably $A D$, given the subsequent $A D$ 353 diagnosis).

\section{Exposure Assessment}

355 High-resolution ambient $\mathrm{PM}_{2.5}, \mathrm{NO}_{2}$, and $\mathrm{O}_{3}$ concentrations at 1-km spatial resolution for the entire United 356 States were derived using spatiotemporal ensemble models that integrated three different machine 357 learning algorithms, including neural networks, random forest, and gradient boosting. The ensemble358 based model was calibrated using hundreds of predictors, including satellite measurements, chemical 359 transport model simulations, land-use terms, meteorological variables, and monitoring measurements 360 from the Environmental Protection Agency (EPA) Air Quality Systems (AQS). This ensemble learning 
approach yielded strong prediction model performance for each pollutant, with an average cross-validated coefficient of determination $\left(\mathrm{R}^{2}\right)$ of $0.89,0.84$ and 0.86 for annual mean $\mathrm{PM}_{2.5}$, annual mean $\mathrm{NO}_{2}$, and

363 warm-season mean maximum 8 -hour $\mathrm{O}_{3}$, respectively ${ }^{34-36}$. We averaged these 1 -km resolution

364 predictions for each pollutant at the ZIP code scale across each year, because ZIP Code is the smallest

365 level of geography in the Medicare data. We used the annual averages in each ZIP code, for each

366 calendar year, as the exposure estimates for each Medicare beneficiary according to the ZIP code of

367 residence. All dementia and AD events were linked to exposures averaged over 5 years prior to

368 diagnosis, and any annual residential mobility changes by ZIP code were taken into account, based on

369 their yearly residence in the Medicare database.

\section{Covariates}

371 Individual-level age at entry, sex, race, and Medicaid eligibility were obtained from the Medicare

372 denominator file. We also obtained neighborhood-level covariates in our study. These included ZIP code-

373 level SES variables (population density, \% Black population, education, median household income, \%

374 owner-occupied housing units, and \% population above 65 years of age living below the poverty line),

375 county-level behavioral risk factors (smoking rate and body mass index) and health care capacity

376 variables (number of hospitals and active medical doctors), as well as a geographical region. Data were

377 also available for co-morbidities (diabetes, heart failure, stroke, hypertension) in CCW. These covariates

378 have been associated previously with ADRD and may be associated with air pollution, and hence were

379 candidate confounders to be included in models ${ }^{41,42}$.

$380 \quad$ Statistical Analysis

381 We fit a series of stratified Cox proportional-hazards models with a generalized estimating equation $382(\mathrm{GEE})^{43}$ to estimate the associations between long-term exposure to $\mathrm{PM}_{2.5}, \mathrm{NO}_{2}$, and $\mathrm{O}_{3}$ on dementia or $383 \mathrm{AD}$ among the elderly, where the coefficient for the exposure variable was the parameter of interest, and 384 years of follow-up was the time scale. Specifically, we fit single-pollutant, bi-pollutant, and tri-pollutant 385 models and estimated hazard ratios (HRs) per interquartile-range (IQR) increase in the 5-year average of 386 the annual $\mathrm{PM}_{2.5}, \mathrm{NO}_{2}$, and warm-season $\mathrm{O}_{3}$ concentrations in the 5 years prior to diagnosis. All three 
387 pollutants are of interest because some prior literature has shown associations between each of them 388 and dementia7,20,22,44. To allow for flexible strata-specific baseline hazard functions, we stratified all 389 models on four individual characteristics, including sex, race (white, black, other), Medicaid eligibility, and 390 1-year categories of age at study entry. To adjust for potential confounding, we included neighborhood391 level SES, behavioral risk factors, and health care capacity variables in our analyses. Potential residual 392 temporal and spatial trends were controlled by respectively including a linear term for calendar years and 393 indicator variables for the geographical region?

394 To assess the shape of the concentration-response $(C-R)$ relationship between each air pollutant and 395 dementia or $\mathrm{AD}$, we respectively fit penalized splines ${ }^{45}$ for $\mathrm{PM}_{2.5}, \mathrm{NO}_{2}$, and $\mathrm{O}_{3}$, adjusting for all covariates 396 included in the tri-pollutant models. To identify subpopulations who might be more vulnerable than others, 397 we assessed potential effect modification by sex, race, Medicaid eligibility, age groups (aged 75+ vs.

398 below 75), and urbanicity (quartiles of population density) on the multiplicative scale by including 399 interaction terms between these potential modifiers and pollutants.

400 Additionally, we estimated the attributable fraction (AF) of dementia and AD cases due to $\mathrm{PM}_{2.5}$ air 401 pollution, for those in the US exposed to an additional IQR of $\mathrm{PM}_{2.5}$ (a difference of $3.2 \mu \mathrm{g} / \mathrm{m}^{3}$ ), beyond 402 current levels in US cities with relatively low exposure (i.e., $7 \mu \mathrm{g} / \mathrm{m}^{3}$, the counterfactual), using results 403 from the multi-pollutant model, and using standard AF calculations when the entire population is exposed 404 (RR-1)/RR (see Steenland and Armstrong 200646).

405 We conducted a series of sensitivity analyses to test the robustness of our main findings. First, we 406 repeated the analyses using a "clean" period of 10 years, i.e., thinking that excluding cases with a 407 diagnosis during their first 10 years of enrollment would increase the probability that we are capturing the 408 first diagnosis and thus more closely estimating disease incidence, albeit at the cost of a smaller number 409 of years of follow-up and cases. Second, using this new subcohort, we assessed alternative exposure 410 time windows by comparing the results using different lags (0-, 1-, 5- and 10-year lags), in which 411 exposure was assigned either as the annual exposure at 10 years prior to case (or the risk set for given 412 cases), or 5 years prior, or 1 or 0 year prior. We posit that if a shorter lag between exposure and disease 413 fits the data best, this would imply an acceleration of an existing process by air pollution, while a longer 
414 lag might indicate the air pollution has an effect in more initial stages of neurodegeneration. Additionally, 415 to evaluate whether the associations we observe can be attributed to comorbidities also linked to air 416 pollution, we additionally adjusted for the comorbidities (including diabetes, hypertension, stroke, and 417 heart failure), and also restricted analyses to subjects without the comorbidities. Finally, we conducted 418 analyses to estimate the effect of possible outcome misclassification in two ways. First, we fit linear 419 regression models for the rate of dementia or AD (events/person-time) with a GEE, which in theory 420 should target an approximately unbiased estimate of the additive effect ${ }^{47}$. Second, we considered the 421 possible effect of outcome misclassification following methods similar to those described by Fox et al. $422(2005)^{48}$. We obtained estimates of misclassification parameters from Taylor et al. (2009) ${ }^{49}$ and adjusted 423 the observed outcomes for each stratum to match up with the expected true values given pre-specified 424 values for sensitivity and specificity for the outcome classification (details provided in Supplemental 425 Material).

426 All computational analyses were run on the Rollins High-Performance Computing (HPC) Cluster at Emory 427 University. R software, version 4.0.2, was used for all analyses. A two-sided $\mathrm{P}<0.05$ was considered 428 statistically significant.

\section{Data availability}

430 Datasets except the Medicare data reported in the current Article are available on request by qualified 431 scientists. The Medicare data (security level 3 data) are not publicly available, due to restrictions of ethical 432 approval requirements for this study and the Health Insurance Portability and Accountability Act (HIPAA) 433 security rule for security.

\section{Code availability}

435 Custom code that supports the findings of this study is available from the corresponding author on 436 request.

\section{Acknowledgements}


438 This study was supported by the HERCULES Center P30 ES019776 and the Goizueta Alzheimer's Disease 439 Research Center (ADRC) of Emory University (P50 AG025688). The authors acknowledge Dr. Joel 440 Schwartz's lab for providing us with access to their estimated air pollution data and acknowledge Jingxuan 441 Zhao for access to the health care capacity data.

\section{Author contributions}

443 L.S. and K.S. designed research and directed its implementation; L.S., H.L., K.S., and R.H.L. analyzed data, 444 L.S., P.L., Y.Z., and H.L. made the figures and tables, L.S., P.L., W.J.R., and J.S. prepared datasets; L.S., 445 K.S., P.L., R.H.L., S.I., H.C., T.W., J.S. and R.J.W. interpreted the results. L.S. and K.S. lead the writing of 446 the manuscript, with input from all authors.

\section{Competing interests}

448 The authors declare no competing interests. 
1 Livingston, G. et al. Dementia prevention, intervention, and care. The Lancet 390, 26732734 (2017).

2 Heron, M. P. Deaths: leading causes for 2017. (2019).

$3 \quad K$ Khachaturian, Z. S., Khachaturian, A. S. \& Thies, W. The draft "National Plan" to address Alzheimer's disease-National Alzheimer's Project Act (NAPA). Alzheimer's \& Dementia 8, 234-236 (2012).

4 Peters, R. et al. Air pollution and dementia: a systematic review. Journal of Alzheimer's Disease 70, S145-S163 (2019).

5 Fu, P. \& Yung, K. K. L. Air pollution and Alzheimer's disease: a systematic review and meta-analysis. Journal of Alzheimer's Disease, 1-14 (2020).

6 van Wijngaarden, E. et al. Neurodegenerative hospital admissions and long-term exposure to ambient fine particle air pollution. Annals of Epidemiology 54, 79-86. e74 (2021).

7 Shi, L. et al. Long-term effects of PM2. 5 on neurological disorders in the American Medicare population: a longitudinal cohort study. The Lancet Planetary Health 4, e557e565 (2020).

8 Smargiassi, A. et al. Exposure to ambient air pollutants and the onset of dementia in Québec, Canada. Environmental Research 190, 109870 (2020).

9 Grande, G., Ljungman, P. L., Eneroth, K., Bellander, T. \& Rizzuto, D. Association between cardiovascular disease and long-term exposure to air pollution with the risk of dementia. JAMA neurology 77, 801-809 (2020).

10 llango, S. D. et al. The role of cardiovascular disease in the relationship between air pollution and incident dementia: a population-based cohort study. International journal of epidemiology 49, 36-44 (2020).

11 Lee, M., Schwartz, J., Wang, Y., Dominici, F. \& Zanobetti, A. Long-term effect of fine particulate matter on hospitalization with dementia. Environmental pollution (Barking, Essex: 1987) 254, 112926 (2019).

12 Mortamais, M. et al. Long-term exposure to ambient air pollution and risk of dementia: Results of the prospective Three-City Study. Environment International 148, 106376 (2021).

13 Nunez, Y. et al. Fine Particle Exposure and Clinical Aggravation in Neurodegenerative Diseases in New York State. Environmental health perspectives 129, 027003 (2021).

14 Sullivan, K. J. et al. Ambient fine particulate matter exposure and incident mild cognitive impairment and dementia. Journal of the American Geriatrics Society (2021).

15 Jack Jr, C. R. et al. Hypothetical model of dynamic biomarkers of the Alzheimer's pathological cascade. The Lancet Neurology 9, 119-128 (2010).

16 Jacob, D. J. Introduction to atmospheric chemistry. (Princeton University Press, 1999).

17 Jerrett, M. et al. Long-term ozone exposure and mortality. New England Journal of Medicine 360, 1085-1095 (2009).

18 Ailshire, J., Karraker, A. \& Clarke, P. Neighborhood social stressors, fine particulate matter air pollution, and cognitive function among older US adults. Social science \& medicine 172, 56-63 (2017).

19 Ailshire, J. \& Brown, L. L. The importance of air quality policy for older adults and diverse communities. Public Policy \& Aging Report 31, 33-37 (2021).

20 Cerza, F. et al. Long-term exposure to air pollution and hospitalization for dementia in the Rome longitudinal study. Environmental Health: A Global Access Science Source 18 (2019).

21 USEPA. Air Quality - Cities and Counties. (2020). 
50022 Chen, H. et al. Exposure to ambient air pollution and the incidence of dementia: A

501

502

503

504

505

506

507

508

509

510

511

512

513

514

515

516

517

518

519

520

521

522

523

524

525

526

527

528

529

530

531

532

533

534

535

536

537

538

539

540

541

542

543

544

545

546

547

548

549

550 population-based cohort study. Environment international 108, 271-277 (2017).

23 Landrigan, P. J. et al. The Lancet Commission on pollution and health. The lancet 391 , 462-512 (2018).

24 Shaffer, R. M. et al. Fine Particulate Matter and Markers of Alzheimer's Disease Neuropathology at Autopsy in a Community-Based Cohort. Journal of Alzheimer's Disease, 1-13.

25 laccarino, L. et al. Association between ambient air pollution and amyloid positron emission tomography positivity in older adults with cognitive impairment. JAMA neurology 78, 197-207 (2021).

26 Younan, D. et al. Particulate matter and episodic memory decline mediated by early neuroanatomic biomarkers of Alzheimer's disease. Brain 143, 289-302 (2020).

27 Calderón-Garcidueñas, L. et al. Long-term air pollution exposure is associated with neuroinflammation, an altered innate immune response, disruption of the blood-brain barrier, ultrafine particulate deposition, and accumulation of amyloid $\beta-42$ and $\alpha-$ synuclein in children and young adults. Toxicologic pathology 36, 289-310 (2008).

28 Cacciottolo, M. et al. Particulate air pollutants, APOE alleles and their contributions to cognitive impairment in older women and to amyloidogenesis in experimental models. Translational psychiatry 7, e1022-e1022 (2017).

29 Levesque, S., Surace, M. J., McDonald, J. \& Block, M. L. Air pollution \& the brain: Subchronic diesel exhaust exposure causes neuroinflammation and elevates early markers of neurodegenerative disease. Journal of neuroinflammation 8, 1-10 (2011).

30 Ranft, U., Schikowski, T., Sugiri, D., Krutmann, J. \& Krämer, U. Long-term exposure to traffic-related particulate matter impairs cognitive function in the elderly. Environmental research 109, 1004-1011 (2009).

31 Maher, B. A. et al. Magnetite pollution nanoparticles in the human brain. Proceedings of the National Academy of Sciences 113, 10797-10801 (2016).

32 Cohen, A. J. et al. Estimates and 25-year trends of the global burden of disease attributable to ambient air pollution: an analysis of data from the Global Burden of Diseases Study 2015. The Lancet 389, 1907-1918 (2017).

33 Goodman, R. A. et al. Prevalence of dementia subtypes in United States Medicare feefor-service beneficiaries, 2011-2013. Alzheimer's \& dementia 13, 28-37 (2017).

$34 \mathrm{Di}, \mathrm{Q}$. et al. An ensemble-based model of PM2. 5 concentration across the contiguous United States with high spatiotemporal resolution. Environment international 130, 104909 (2019).

35 Di, Q. et al. Assessing NO2 Concentration and Model Uncertainty with High Spatiotemporal Resolution across the Contiguous United States Using Ensemble Model Averaging. Environmental science \& technology 54, 1372-1384 (2019).

36 Requia, W. J. et al. An ensemble learning approach for estimating high spatiotemporal resolution of ground-level ozone in the contiguous United States. Environmental Science \& Technology 54, 11037-11047 (2020).

37 Kioumourtzoglou, M.-A. et al. Exposure measurement error in PM 2.5 health effects studies: a pooled analysis of eight personal exposure validation studies. Environmental Health 13, 2 (2014).

$38 \mathrm{Wu}, \mathrm{X}$. et al. Causal inference in the context of an error prone exposure: air pollution and mortality. The Annals of Applied Statistics 13, 520-547 (2019).

39 Zeger, S. L. et al. Exposure measurement error in time-series studies of air pollution: concepts and consequences. Environmental health perspectives 108, 419-426 (2000).

$40<$ https://www2.ccwdata.org/web/guest/condition-categories $>$ (

41 Hersi, M. et al. Risk factors associated with the onset and progression of Alzheimer's disease: A systematic review of the evidence. Neurotoxicology 61, 143-187 (2017). 
42 Anstey, K. J., Ee, N., Eramudugolla, R., Jagger, C. \& Peters, R. A systematic review of meta-analyses that evaluate risk factors for dementia to evaluate the quantity, quality, and global representativeness of evidence. Journal of Alzheimer's Disease 70, S165S186 (2019).

43 Zhang, X. Generalized estimating equations for clustered survival data. (2006).

44 Chen, H. et al. Living near major roads and the incidence of dementia, Parkinson's disease, and multiple sclerosis: a population-based cohort study. The Lancet 389, 718726 (2017).

45 Meyer, M. C. Constrained penalized splines. Canadian Journal of Statistics 40, 190-206 (2012).

46 Steenland, K. \& Armstrong, B. An overview of methods for calculating the burden of disease due to specific risk factors. Epidemiology, 512-519 (2006).

47 Hutcheon, J. A., Chiolero, A. \& Hanley, J. A. Random measurement error and regression dilution bias. Bmj 340 (2010).

48 Fox, M. P., Lash, T. L. \& Greenland, S. A method to automate probabilistic sensitivity analyses of misclassified binary variables. International journal of epidemiology 34 , 1370-1376 (2005).

570

49 Taylor Jr, D. H., Østbye, T., Langa, K. M., Weir, D. \& Plassman, B. L. The accuracy of Medicare claims as an epidemiological tool: the case of dementia revisited. Journal of Alzheimer's Disease 17, 807-815 (2009). 


\section{Supplementary Files}

This is a list of supplementary files associated with this preprint. Click to download.

- Supplementarymaterials.docx 